J.J. Ramsden, A.A. Mamali, A.G. Mamalis and N.T. Athanassoulis

\title{
Nanotechnology and sustainability
}

ABSTRACT. The sustainability of our present civilization and, ultimately, of human life itself is challenged on many fronts. The most prominent of the challenges are climate change, extreme food and water shortages, rising chronic diseases, and rampant obesity. They are all of great significance in terms of death and morbidity, and at the same time seemingly intractable. This paper looks at the technical dimension of overcoming these challenges, contrasting the apparent impotence of conventional technologies with the potential of nanotechnology. Attention is paid to the scalability of any proposed nanotechnology-based solutions, as well as the related aspect of realizable timescales. The paper concludes by examining the problems of implementing solutions projected to be successful.

Nanotechnology Perceptions 14 (2018) 159-166

doi: 10.4024/N18RA18A.ntp.14.03 